\title{
CHEMOTHERAPY OF NON-PULMONARY TUBERCULOUS CONDITIONS
}

By Peter H. Buxton, M.R.C.P. From the Middlesex Hospital, London

Many drugs have been tested for their effect in vitro on the growth of the tubercle bacillus; some of these have been tested in experimental tuberculosis in animals and some have been given clinical trials (Feldman, 1946). By far the most effective yet to have had extensive clinical trials is streptomycin. Apart from the toxic effects of this drug on the vestibular and auditory apparatus, its value was limited by the rapid development of drug resistence by the tubercle bacillus. At first attempts were made to combat this by giving intermittent treatment; they were not successful. In 1949 the Medical Research Council were able to show, as a result of clinical trials then proceeding with paraamino salicylic acid. (PAS) and streptomycin in pulmonary tuberculosis, that if PAS was given concurrently with streptomycin therapy there was a considerable reduction in the incidence of streptomycin resistant strains of tubercle bacilli produced; this has since been confirmed by many other workers. It is now general practice to give PAS by mouth in all cases of tuberculous infection that are treated by streptomycin systemically.

The dosage of PAS usually recommended is $20 \mathrm{~g}$. per day for adults, given in divided doses four times a day, but it is wise to start with a smaller dosage, such as $12 \mathrm{~g}$. per day, which should be increased gradually. Side effects of the drug are anorexia, nausea and vomiting, which tend to occur at the start of treatment. Different forms of the drug have been produced in an attempt to counteract these side effects; our experiènce has been that the granule form is that best tolerated and that if the dose is increased slowly, digestive disturoances usually subside as treatment is continued.

The place of other antibiotics and antibacterial substances introduced for the treatment of tuberculosis has recently been summarized by Scadding (I95I).

\section{Treatment of Tuberculous Lymphadenitis}

General measures such as bed rest and sanatorial treatment are still necessary in the active stage of the disease.

Chemotherapy alone with intramuscular streptomycin and oral PAS is the treatment of choice for extensive involvement of glands in the exudative phase. Surgical removal is the best treatment for caseating glands or for localized groups of infected glands.

When chemotherapy alone is used, streptomycin should be given intramuscularly in the dosage of I g. per day for adults and ro mg. per lb. body weight per day for children; this dose is usually divided into two injections given night and morning. PAS should be given orally concurrently with streptomycin treatment. Treatment is continued till there is no further evidence of activity as - judged by the temperature, sedimenţation rate, reduction in size and tenderness of glands and gain of weight. If after 12 weeks' treatment there is still evidence of activity, it is better to discontinue all drugs and observe the patient for two weeks, as occasionally persistent pyrexia may be due to streptomycin or PAS. If, however, the patient's condition continues to deteriorate during this rest period, a further course of streptomycin and PAS should be given. In the treatment of widespread tuberculous adenitis the use of streptomycin and PAS in conjunction with general measures has shortened considerably the course of the disease. In some cases the temperature response and improvement in the patient's general condition have been dramatic.

When chemotherapy is given as a cover for surgery in the presence of active disease, treatment with intramuscular streptomycin and oral PAS in the dosage mentioned above should be started at least 24 hours before operation and continued for at least a week after operation, and $0.5 \mathrm{~g}$. of 
streptomycin either dry or in solution should be applied locally at the time of operation. Using this technique a very high percentage of operation wounds heal by first intention, leaving minimal disfigurement.

In cases of cold abscess treatment is by aspiration and replacement with streptomycin solution, $0.5 \mathrm{~g}$. in I ml. distilled water. Surgical removal of the infective focus may be required later, and it is wiser, if this is planned, to reserve systemic streptomycin treatment till then.

\section{Treatment of Bone and Joint Tuberculosis}

There is no large series of cases yet published in this country. showing the long-term results of streptomycin treatment in bone and joint tuberculosis. Since the advent of streptomycin no major change has taken place in the principles of treatment laid down by Girdlestone (1940) and Mercer (1950). Importance is still attached to general measures such as bed rest, sunlight and fresh air. Immobilization of the affected part and reduction of the deformity are the main aims of treatment; in adults excision of the infected joint or arthrodesis are often necessary. Treatment with streptomycin and PAS has not produced startling improvement in the actual bony disease, but it has been impressive in reducing cachexia and allowing tuberculous sinuses to heal.

In joint tuberculosis, Streeten (1949) claimed rapid improvement and relief of pain with cure and useful mobility in the synovial type of disease, by preliminary alkalinization of the joint space with sodium hydroxide and borax buffer solution; streptomycin 0.2 to $0.5 \mathrm{~g}$. was then injected daily into the joint cavity. Unfortunately the follow-up period in his cases was too short to give a final assessment of the method.

At present most workers use the classical methods of treatment outlined above with, in addition, intramuscular streptomycin I g. per day and oral PAS $20 \mathrm{~g}$. per day. Should tuberculous sinuses arise, local streptomycin is given as well. Cold abscesses should be treated conservatively if possible; aspiration and replacement with streptomycin solution may be used but incision should be reserved for cases where the skin is discoloured and about to break, or the abscess is spreading, or where there is pressure on vital parts or secondary infection. In all cases of secondary infection the infecting organism should be isolated and the appropriate antibiotic given after in vitro sensitivity tests.

As in cases of tuberculous adenitis there is no dramatic cure of the underlying disease with streptomycin therapy, but tuberculous sinuses can be expected to close and cachexia is relieved.

\section{Tuberculous Sinuses}

Tuberculous sinuses may be treated with streptomycin given locally (Ahern, 1950), or the drug may be given, systemically with oral PAS (Davis, 1949). The adherents of local treatment alone argue that their results as regards closure of the sinuses are as good as when systemic treatment also is given, but that their method has the advantage of saving the patient much discomfort from repeated intramuscular injections. They also state that though the drug is only given locally, therapeutic blood levels are obtained and the concentration of the drug at the site of the infection is greater than that obtained by intramuscular treatment alone. There is, however, always a greater chance of producing drug sensitivity when any antibiotic drug is administered in large doses locally, and there is also the danger that the drug may not penetrate through dense granulation tissue and necrotic débris in sufficiently high concentrations to exert a bacteriostatic effect on deepseated viable tubercle bacilli. If a deep focus of infection is suspected, it is better to treat this either by systemic streptomycin and PAS, or by surgery, the sinus itself being treated by local applications of streptomycin.

Results of streptomycin treatment in closure of sinuses are excellent, but there is always a danger of breakdown unless the underlying infection can . be removed or controlled.

\section{Treatment of Tuberculous Peritonitis}

Only three cases of tuberculous peritonitis treated with streptomycin have been reported in this country, and in all the results were most successful (Grant et al., 195I; Salmon, r95I). Scarcity of puolished reports is probably due to the fact that individual centres have only had occasional cases to treat. At the Middlesex Hospital we have treated three cases. One, after a severe emotional shock, developed tuberculous meningitis and died, but the other two are apparently cured, though the period of follow-up is only six months.

Treatment in our cases and the other three reported cases was by streptomycin, I g. per day intramuscularly, with PAS by mouth. The total dose of streptomycin was 80 to $100 \mathrm{~g}$. Salmon stresses the importance of good nursing and attention to diet. The dramatic response to treatment in his case is shown by the fact that $2 \frac{1}{2}$ years after the onset of her illness, the patient completed a normal pregnancy and delivered herself of a normal full-term child.

\section{Treatment of Tuberculous Meningitis}

Streptomycin is the most potent drug yet discovered for the treatment of tuberculous meningitis. 
Since it was introduced the average survival rate has been approximately $I$ in 4 , though individual centres have reported survival rates of 50 per cent. The most reassuring feature is the high percentage of survivors classified as clinically well after two to three years' observation (Ministry of Health, I950).

Most workers are agreed that the most important single factor affecting prognosis is the severity of the disease at the start of treatment. The early diagnostic features have been well described by Rubie and Mohun (1949).

\section{Pathology}

A brief account of the pathology of the disease is given as it has an important bearing on the treatment and complications. At post-mortem in cases of tuberculous meningitis the two characteristic findings are a widespread arteritis, and a gelatinous exudate most marked over the base of the brain. The arteritis leads to thrombosis and cerebral softening, and often accounts for the sudden onset of focal signs. The exudate, which in time is converted into fibrin, accounts for the blocks that arise in the cerebrospinal fluid pathways.

The sites of these blocks, shown in Fig. I, are remarkably constant and are probably due partly to the varying rates of flow at different points, and partly to the supine position in bed and immobility of the patient so typical of the disease. The commonest sites of obstruction are the cisterna chiasmatica and the transverse fissure, blockage at these sites producing a communicating hyarocephalus. The collection of exudate at the cisterna chiasmatica may also account for the pituitary or hypothalamic disorders not infrequently encountered during treatment. Exudate and adhesions in the region of the transverse fissure may cause obstruction to the venous return from the choroid plexuses by blocking the great vein of Galen, so leading to an enormous increase in intraventricular pressure.

Apart from the dangers of obstruction, this exudate also prevents the access of streptomycin to viable tubercle bacilli.

\section{Treatment}

General measures are of the utmost importance in determining the successful outcome of treatment. Bed rest in the active stage of the disease, good nursing, a nutritious diet and meticulous care over intrathecal therapy are essential.

Intramuscular streptomycin. Most workers are now agreed that streptomycin should be given intramuscularly twice daily for the first six months of treatment at least. A total dose of 2 g. per day

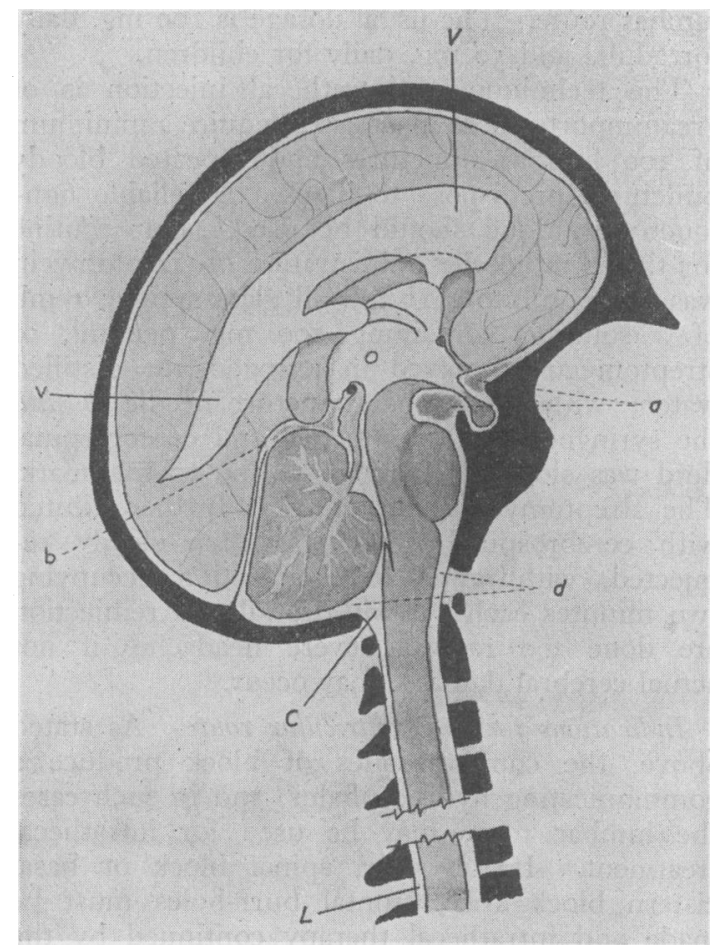

Schematic diagram of cerebrospinal fluid pathway showing routes for intrathecal therapy; L. lumbar C. cisternal; V. ventricular. Sites where exudat collects: a. cisterna chiasmatica; b. transvers fissure; $d$. basal cisterns.

is given for adults and $20 \mathrm{mg}$. per $\mathrm{lb}$. body weight per day for children. A dose of $1 \mathrm{~g}$. per day has been suggested in order to reduce the incidence of damage to the eighth nerve, but one of our cases failed to respond to the smaller dose and as vestibular damage following treatment of this disease with streptomycin is almost inevitable, it seems unjustifiable in such a lethal infection not to use the larger dose. On intramuscular streptomycin, $2 \mathrm{~g}$. per day, levels of 4 to 16 microg. are found in the spinal fluid, depending on the activity of the meningitis. In the early days of streptomycin therapy some workers considered that intrathecal treatment was unnecessary as bacteriostatic levels of streptomycin were found in the spinal fluid on intramuscular therapy alone. However, a report from the Medical Research Council in 1948 showed that cases given intramuscular treatment alone did not respond 80 well as those in which the drug was also given intrathecally.

Intrathecal treatment. In cases uncomplicated by blockage of the cerebrospinal fluid pathways, intrathecal treatment should be given by the 
lumbar route. The usual dosage is $100 \mathrm{mg}$. daily for adults and $50 \mathrm{mg}$. daily for children.

The technique of intrathecal injection is of great importance as most cases require a minimum of roo lumbar punctures and repeated bloody punctures predispose to block. A reliable nontouch technique should be used. Our routine for the intrathecal administration of streptomycin was to draw into a $10 \mathrm{ml}$. all glass syringe $\mathrm{r} \mathrm{ml}$. of a solution containing roo $\mathrm{mg}$. per $\mathrm{ml}$. of streptomycin dissolved in pyrogen-free distilled water; with the lumbar puncture needle in situ, the syringe was then attached and cerebrospinal fluid was slowly withdrawn to the ro $\mathrm{ml}$. mark. The streptomycin solution, thus further diluted with cerebrospinal fluid, was then slowly reinjected, withdrawal and reinjection occupying two minutes each; if withdrawal and reinjection are done too rapidly severe headache, if not actual cerebral damage, may occur.

Indications for the ventricular route. As stated above, the common sites of block produce a communicating hydrocephalus, and in such cases the lumbar route may be used for intrathecal treatment. lf, however, spinal block or basal cistern block arise, frontal burr-holes must be made and intrathecal therapy continued by the intraventricular route.

The diagnosis of spinal or basal cistern block is difficult clinically, though it usually occurs in the more severe cases and is associated with severe headache and vomiting; papilloedema may or may not occur. The cerebrospinal fluid findings are, however, the best guide. A rising protein figure, particularly if this is over $600 \mathrm{mg}$. per roo $\mathrm{ml}$., and increasing difficulty in withdrawal of the lumbar fluid associated with no rise in pressure of the fluid on compression of the jugular veins, are indications of spinal block. Absolute block is indicated by either a dry tap or the removal of a few drops of xanthochromic fluid of high protein content. Once such a block is suspected burrholes should be made and the degree of obstruction assessed by taking simultaneous lumbar and ventricular fluid pressures while the patient is tilted.

The dose of streptomycin recommended for intraventricular therapy is $50 \mathrm{mg}$. per day for adults and $25 \mathrm{mg}$. per day for children, alternate ventricles being used on successive days. If the intraventricular pressure is found to be raised, this should be reduced to normal by withdrawal of cerebrospinal fluid before the introduction of streptomycin. Ventricular treatment should be continued till there is resolution of spinal block. When this occurs treatment is continued by the lumbar route.

Cisternal puncture is seldom of value in the diagnosis of block and is not practicaole in an ill patient for daily intrathecal treatment.

Direct instillation of streptomycin into the sites where maximum exudate is known to arise-the chiasmal region and interpeduncular space-has been attempted. Such treatment requires extensive neurosurgery, and is now considered to have little place in the management of the disease, as by the time indications for this treatment arise, dense adhesions and widespread arteritis are already present.

Duration of intrathecal therapy. As individual prognosis is difficult and occasionally cases with gross neurological signs make a good functional recovery, treatment should not be withheld till the stage of decerebrate rigidity is reached (Smith and Vollum, 1950). The risks of saving a life with residual mental defect are usually over-emphasized, except perhaps in the case of young infants (Illingworth and Lorber, I95I).

It is generally agreed that daily intrathecal treatment should be given for at least the first six weeks. After this initial period opinions differ as to the intensity of treatment necessary. Whatever general scheme is adopted this must be modified in the light of clinical and laboratory findings. It must be stressed that persistent headache, pyrexia, development of focal signs and continued loss of weight, are most likely to be due to activity of the disease rather than to over-zealous treatment. When the clinical signs mentioned are assessed, consideration must be given to tuberculous lesions outside the central nervous system as well as within it. The most reliable guides to progress in the spinal fluid are the cell count and the sugar content. A raised lymphocyte count usually indicates activity of the disease; though strepto-

mycin itself may cause a rise in cells, these are usually predominantly polymorphonuclears. A falling sugar figure in the fluid is very suggestive of activity of the disease. Intrathecal therapy should be continued while there is still clinical evidence of activity of the disease in the central nervous system, or if the spinal fluid contents remain grossly abnormal. As improvement takes place clinically and in the laboratory findings, intrathecal injections may be reduced in frequency, but they should not be stopped entirely till there is no clinical evidence of activity, till the sugar and chloride contents of the spinal fluid have risen to within 10 per cent. of normal, and till the cells are less than 20 per $\mathrm{cmm}$. When this stage is reached intrathecal therapy is stopped, but intramuscular therapy is continued for at least a month. Weekly lumbar punctures should be done when intrathecal treatment is stopped to confirm that improvement is maintained. At the weekly examinations of the spinal fluid the streptomycin 
content of the fluid is estimated. Levels of more than 2 microg. per ml. on intramuscular treatment alone indicate breakdown of the blood-brain barrier, strongly suggesting activity of the meningitic process. The protein content of the spinal fluid is of little value in estimating activity of the disease as it may take months to return to normal after the patient has otherwise recovered completely. If improvement is maintained when intrathecal therapy has been stopped for a month, intramuscular treatment may be discontinued. After a further period of observation in hospital, adults should be sent to convalescence, and children, if practicable, to a convalescent school. After streptomycin treatment is stopped, monthly clinical and lumbar puncture examinations should be carried out to check progress, and if this is maintained the intervals between examinations can be progressively lengthened.

Damage to the vestibular apparatus occurs in nearly all cases, and is no indication for withholding treatment. Survivors under the age of $5^{\circ}$ years are left with little residual disability owing to the development of other compensating mechanisms. One of our survivors, a child with absent labyrinthine response to ice water, successfully learnt to skate.

Deafness is not considered to be an indication for stopping treatment. Once it has occurred, it is doubtful whether recovery will take place even if streptomycin treatment is stopped immediately.

\section{Additional drug treatment}

Para-amino salicylic acid should be given orally to all cases concurrently with streptomycin therapy.

Sulphetrone has been popular on the Continent as an adjuvant to streptomycin treatment. In this country results after giving the drug by mouth have been unconvincing, and it is considered that severe toxic effects occur too frequently to justify its routine use orally. It has been used intrathecally, but again with inconclusive results (Cathie and MacFarlane, 1950).

Streptokinase is an enzyme obtained from Streptococcus pyogenes which will lyse fibrinous exudate in vitro. It has been used intrathecally in an attempt to prevent adhesions and block (Cathie, I949). Lorber, assessing the value of the drug with a control series of cases, has recently shown that its efficacy is very doubtful. As it may itself produce meningeal irritation the use of the enzyme is probably no longer justifiable.

Intrathecal tuberculin. Smith and Vollum (1950) have suggested that one of the factors in tuberculous meningitis restricting the survival rate, and perhaps explaining the occurrence and disappear- ance of block, is the host response to tuberculoprotein. They showed that if minute amounts of purified protein derivative (PPD) were injected into the subarachnoid fluid of patients with tuberculous meningitis, a very brisk meningeal reaction occurred. When this had subsided they found, surprisingly, clinical improvement in cases previously considered hopeless. They also found later evidence of resolution of exudate previously present.

It may be that the use of intrathecal PPD will mark an advance in the treatment of tuberculous meningitis, though results in a recent paper by Fletcher (195I) were not so promising. 1t was stressed by Smith and Vollum that intrathecal PPD treatment was potentially extremely dangerous, and it would seem wise till further information is available to reserve it for severe cases deteriorating despite intensive streptomycin therapy.

\section{Summary and Conclusions}

Present trends in the treatment of some nonpulmonary tuberculous conditions are discussed. The main principle of treatment is to bring an adequate concentration of streptomycin to the site of the infection. This is usually best attained by the blood stream. The drug should therefore be administered intramuscularly except in superficial infections where local treatment alone may suffice. In cases of tuberculous meningitis it is necessary to attain a high concentration of streptomycin in the cerebrospinal fluid so that the drug may diffuse through exudate to reach the underlying infection in bacteriostatic concentrations, thus intrathecal treatment is necessary in these cases as well as intramuscular. The management of intrathecal block is discussed.

The routine addition of PAS to streptomycin treatment has reduced the incidence of streptomycin resistant tubercle bacilli found in treated cases. This is important not only for the patient undergoing treatment but also for contacts who may be infected by him.

Treatment with streptomycin and PAS offers hope of cure in cases of tuberculous meningitis, a previously fatal disease, and has improved the prognosis and shortened the course of other tuberculous infections. It is stressed, however, that general measures and good management are still of the utmost importance in attaining successful results.

\section{BIBLIOGRAPHY}

AHERN, R. T. (1950), Lancet, i, 443.

CATHIE, I. A. B. (1949), Lancet, i, 44I.

CATHIE, I. A. B., and MACFARLANE, J. C. W. (1950), Ibid., ii, 784 .

DAVIS, R. H. (1949), Ibid., ii, 982. 
FELDMAN, W. H. (1946), 'Harben Lecture,' F. Rqy. Inst. Pub. Health \& Hygiene, 9, 267, 297, 343.

FLETCHER, A. P. (1951), Lancet, ii, 290.

GIRDLESTONE, G. R. (1940), 'Tuberculosis of Bone and Joint,' Oxford Univ. Press, Oxford.

GRANT, R. A., GRUNBERG, A., and LINDSAY BLAIR, J. (I95I), Brit. med. f., i, 740 .

ILLINGWORTH, R. S., and LORBER, J. (1951), Lancet, ii, $5 \mathrm{II}$. LORBER, J. (1951), Ibid., i, 1334.

MEDICAL RESEARCH COUNCIL (1948).
MERCER, W. (1950), 'Orthopaedic Surgery,' Arnold, London. MINISTRY OF HEALTH STREPTOMYCIN TRIALS (1950), 'Meningitis and Miliary Tuberculosis,' H.M. Stationery Office, London.

RUBIE, J., and MOHUN, A. F. (1949), Brit. med. F., i, 338.

SALMON, H. W. (195I), Lancet, ii, 153.

SCADDING, J. G. (195I), Postgrad. med. F., 27, 549.

SMITH, H. V., and VOLLUM, R. L. (1950), Lancet, ii, 275.

STREETEN, D. H. P. (1949), South African med. Y., 23, 777, 850.

\section{The Comfort Crooksbank Award for Cancer Researcb}

The Comfort Crookshank Award for Cancer Research was awarded at the Middlesex Hospital, on October 8, to Dr. J. J. Bittner. After receiving the award he delivered a lecture entitled 'The Genesis of Mammary Cancer in Mice.' He described experiments which had been performed with strains of mice which had been maintained for more than 20 generations by brother-to-sister mating. Some of these strains were infected with the 'milk agent' which is responsible for some mammary carcinomata in those mice hereditarily susceptible to its influence. Suspensions of the agent, when diluted within limits, produced a higher incidence of cancer than when undiluted. With repeated doses a smaller incidence of cancer was obtained than with single doses in other mice and their progeny. These last two facts suggest that an active immunity could be developed against the milk agent. Tumour material which had been frozen and dried produced tumours at the site of inoculation in those mice which would support growth of transplants of fresh tumour materialmore tumours were produced by intraperitoneal than by subcutaneous injections. Evidence showed that male mice had infected females with the milk agent.

Dr. Bittner showed pictures of sections of whole mammary glands in which there were hyperplastic nodules said by some to be precancerous and to be associated with those breasts susceptible to the milk agent. He stated that some were of the opinion that the degree to which breasts produced hyperplastic nodules in response to artificially administered oestrogens was directly proportional to their susceptibility to the milk agent. This observation, together with further evidence ad-o duced from experiments on various strains of mices in which ovariectomy and transplantation of ovaries and adrenals were carried out, led to the hypothesis that the milk agent was responsible for altering the hormone balance of mice infected with it. Finally, in support of this hypothesis there was the striking fact that those mice infectedo by the milk agent excreted a smaller amount of r7-ketosteroids than those free of agent. Dr. Bittner said it was possible that alteration of the hormone balance was directly responsible for the production of mammary cancer in mice, and that the role of the milk agent might merely be that of upsetting the hormones.

In mice there occur mammary adenocarcinomata, both spontaneous and chemically induced, which are not associated with the milk agent; thus, arguing by analogy, it is possible that there are several kinds of human breast cancer one of which may be transmitted by a milk agent. If this is so, it must be borne in mind that human females could be infected from the male or by such things as blood transfusion. However, 'Dr. Bittner calculated that it would be at least 80 years before it could be determined whether a milk agent played any part in human breast cancer. 